\title{
ANOTHER PROOF OF THE DENUMERABILITY OF THE COMPLEX NUMBERS
}

\author{
J. Ulisses Ferreira \\ Trv. Pirapora 36 Costa Azul 41.770-220 \\ Salvador, Brazil \\ ulisseseferreira.mat.br
}

\begin{abstract}
This short paper suggests that there might be numerals that do not represent numbers. It introduces an alternative proof that the set of complex numbers is denumerable, and also an algorithm for denumerating them.
\end{abstract}

\section{KEYWORDS}

Enumeration, Georg Cantor, diagonal process

\section{INTRODUCTION}

In 2004 [1] and 2005 [2], I demonstrated that C, i.e. the set of the complex numbers, is denumerable. Before that, it was already known that $\mathrm{Q}$, i.e. the rational numbers, is denumerable, and that repetitions of the same numbers, such as 1/2,2/4 and 3/6 in any denumeration such as

$$
0,1 / 1,-1 / 1,1 / 2,-1 / 2,2 / 1,-2 / 1,1 / 3,-1 / 3,2 / 2,-2 / 2,3 / 1,-3 / 1 \ldots
$$

caused no problem. The order of the numbers does not matter either. Furthermore, I observed that the Cantor's diagonal process was based on numerals, instead of numbers.

One cannot arbitrarily invent any set of new numbers. Historically, the zero and negative integers were discovered because there was some need: the subtraction operation. Then, the rational numbers were discovered since there was the need for dividing numbers, and so on. Thus, except for the natural numbers, the notion of number expresses quantity but it is also the result from a sequence of arithmetical operations. Pi appeared because of the geometry, and so on. Thus, there do exist irrational numerals between 0 and 1 that do not represent numbers, possibly most of them, if we can talk in this way. This means that using randomizing functions such as the well known randomize and rand in a programming language, even if the computer memory was infinite, will produce numerals based on decimal digits, but not necessarily numbers. Since the memory is finite, they do not even produce irrational numbers in such a representation. Not even $10 / 3$ can be represented on a computer using decimal digits only.

Cantor's diagonal process just seems to work because it was a deduction over symbols.

Dhinaharan Nagamalai et al. (Eds) : CSE, DBDM, CCNET, AIFL, SCOM, CICS, CSIP - 2014

pp. 79-82, 2014. (C) CS \& IT-CSCP 2014

DOI : $10.5121 /$ csit.2014.4407 
In [1,2], I also proved that not only $\mathrm{R}$ but also $\mathrm{C}$ are denumerable. In this short paper, I introduce a proof that is easier to be understood.

Note that MMXII (Roman) and 2012 (Arabic) are two different numerals that represent the same number, and that this is much older than Cantor and our culture. Whether there are or not numerals in decimal Arabic notation that are supposed to represent some irrational number but do not represent any number, the following proof works.

\section{The Proof}

Here, I use the coding and decoding system previously used by Kurt Gödel in the proof of his historic theorem of incompleteness, published in 1931 [3], which, in its turn, was inspired by Whitehead and Russell's work entitled Principia Mathematica [4]. The used numbers are better known as "Gödel Numbers".

As a professional originally from the area of programming languages design and implementation, whenever I do not have suitable means for representing any arithmetic expression, I simply write the expression as if I was using a subset of some full programming language, such as BASIC, Pascal, Java, Haskell or any other, taking into consideration that I use the symbol " $\wedge$ " to represent power like in BASIC, instead of "**" like in FORTRAN and that some languages do not provide the power function. In this way, those are the numerals that I chose for representing the set $\mathrm{C}$ of the complex numbers: for instance, I represent the constant $e$ by simply "e"; For the irrational number $\pi$, I simply represent it by "pi"; The irrational number which is the square root of -1 , I represent by "sqrt(-1)", but as I wish to also represent all complex numbers, I simply write " $\mathrm{i}$ " for representing the same number, and so on. A few examples of numerals of mine are "10/3", "sqrt(2)", “ $1+3 /(\sin (30) * 2) ", " \log (2,10)-$ pi", “e^32", "2+6*i”, each of them representing its corresponding complex number. Furthermore, since they are arithmetic expressions only, not logical propositions nor predicates nor function definitions, there is no self-references or paradoxes involved.

Given a coding table for characters such as ASCII, the next step consist in calculating the Gödel numbers that correspond to those strings, using a function that I would naturally define having $\mathrm{G}$ as its identifier. Let $c$ be the function that receives a character and results in its ASCII code (or, alternatively, any more suitable code from a shorter table), and, skipping 1, let us use the same sequence of the first smaller prime numbers such as the one used by Gödel while proving that theorem of his, I obtain the following examples:

$\mathrm{G}\left(\right.$ ("e") $=2^{\wedge} \mathrm{c}($ 'e').

$\mathrm{G}\left(\right.$ ("pi”) $=2^{\wedge} \mathrm{c}\left(\right.$ 'p’) * $3^{\wedge} \mathrm{c}($ 'i’).

$\left.\mathrm{G}\left({ }^{\prime} 10 / 3^{\prime}\right)=2^{\wedge} \mathrm{c}\left({ }^{\prime} 1 ’\right) * 3^{\wedge} \mathrm{c}\left({ }^{\prime} 0 ’\right) * 5^{\wedge} \mathrm{c}\left({ }^{\prime} /\right)\right) * 7 \wedge \mathrm{c}\left({ }^{\prime} 3^{\prime}\right)$.

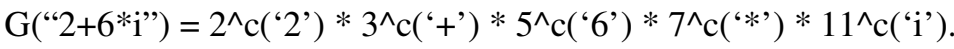

One can observe that there are infinite Gödel numbers that do not represent any complex number, but this is not a problem since both sets are infinite and any subset of the natural numbers is denumerable.

Since the function $\mathrm{G}$ always receives a complete expression as a string and results in a natural number, and, using the numerals that I chose as strings containing arithmetic expressions, I can represent any complex number, the set of the complex numbers is denumerable. 
Remark: Note that, as in any coding function using Gödel numbers, there is an inverse function that, from any natural number obtained from the above explained manner, results in the string that contains the original arithmetic expression.

Theorem 2. There does exist some algorithm that denumerates the set of the complex numbers. Proof. I will prove constructively, showing my algorithm: first, I define an alphabet, a formal language for arithmetic expressions and write a parser for expressions of this language, which, in its turn, contains only the alphabet defined for constant expressions, operators and symbols such as "(", "," and ")" used in expressions, identifiers of all arithmetic functions such as "log" and "sin", and reserved words of constant values, such as "e", "pi" and "i", but that language does not contain any variable. One typically uses BNF for doing so.

A question is to know what the first number of my denumeration is. Thus, I have to set an order. Let G' be the inverse function that receives a Gödel number and results in the original arithmetic expression. Starting from the natural number $n=2$, we apply $G$ ' and verify to know whether the resulting string denotes any well-formed arithmetic expression, in accordance with the formed language previously defined. If true, this is the first numeral of my denumeration. Otherwise, we can try using $n=3$, then $n=4$, then $n=5$, and so on, until we find the first natural number $n$ whose $G^{\prime}(n)$ application results in some arithmetic expression well formed. The obtained string is the first numeral, which, in its turn, represents the first number of my denumeration.

Moreover, we obtain the other numbers in order using the following algorithm, in Algol 60 or Pascal style:

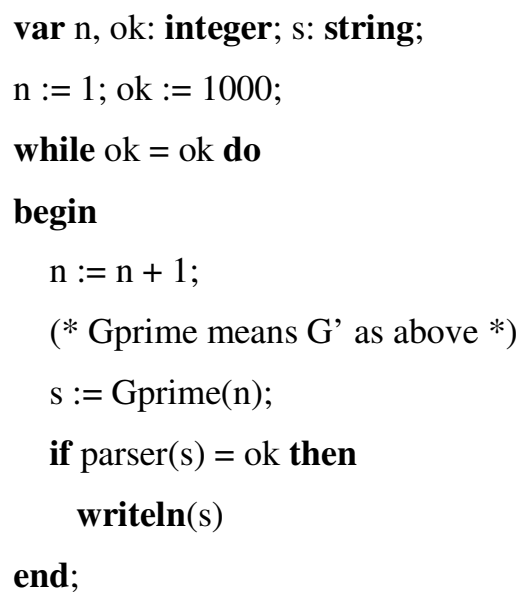

In the end, what is written by the above algorithm is a sequence of complex numbers, i.e. a denumeration of them.

\section{Conclusions}

There are no transfinite numbers, and there is no set with cardinality greater than infinite. Cantor, Post and others collapsed the older notions of numbers and numerals, and that was a mistake. There is no need for the notion of "computable numbers" either, since what was called "computable number" is just a number whose numeral in the Arabic decimal notation can represent another number up to some given precision. 


\section{REFERENCES}

[1] Ferreira, Ulisses, (2004) "The real set can be seen as denumerable", In Hamid R. Arabnia, Iyad A. Ajwa, and George A. Gravanis, editors, Post-Conference Proceedings of the 2004 International Conference on Algorithmic Mathematics \& Computer Science, CSREA Press, Las Vegas, Nevada, USA, pp523-526.

[2] Ulisses Ferreira (2005) The sets of real and complex numbers are denumerable. ACM SIGACT News 36(2): pp126-130.

[3] Gödel, Kurt (1931) Uber formal unentscheidbare sätze der principia mathematica und verwandter system i. Monatschefte Mathematik und Physik. 38, pp173-98.

[4] Whitehead, Alfred \& Russell, Bertrand (1910) Principia Mathematica, Cambridge University Press, 3 volumes, 13 .

\section{Author}

Ferreira was born in Salvador in 1961, studied and did some research work in computer science at UFBA, UFPB, Sussex University, Edinburgh University and Trinity College in Dublin.

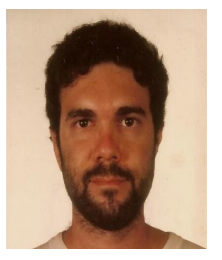

